\section{Strontium-calcium substitution in synaptic transmission in turtle retina}

RECENT electrophysiological and morphological studies strongly suggest that the synaptic transmission between photoreceptors and horizontal cells in the vertebrate retina is chemical in nature, although the anatomical appearance of the neuronal contacts does not strictly conform to that of conventional chemical synapses ${ }^{1-3}$. It has been shown that changes in the ionic composition of the extracellular medium, such as lowering calcium, increasing magnesium or adding cobalt, that in the chemical synapses are known to block the release of transmitter ${ }^{4,5}$, abolish the response to light of horizontal cells without reducing that of photoreceptors ${ }^{6-8}$. The present report provides additional evidence that the synapses between photoreceptors and horizontal cells have properties characteristics of conventional chemical synapses. It is known that in the chemically transmitting synapse, strontium ions can substitute calcium ions in the process of transmitter release ${ }^{9-11}$. I have studied the effects of calcium-strontium substitution on the intracellularly recorded responses to light of horizontal cells and cones in the retina of the turtle (Pseudemys scripta elegans).

After draining the vitreous humour, the isolated eyecup, with the retina still attached to the pigmented epithelium, was mounted in a perfusion chamber ${ }^{12}$ where a modified Ringer solution continuously flowed over the vitreous side ${ }^{13}$. Intracellular recordings were made with glass micropipettes filled with $4 \mathrm{M}$ potassium acetate. The light stimuli were the reduced images of circular apertures, the diameter of which on the retina could be varied from 1,200 to $100 \mu \mathrm{m}$. The light source was a quartz iodine lamp (Riluma $100 \mathrm{~W}$ ) and neutral density filters were used to reduce light intensity. The total flux density of unattenuated light at retinal level was about $1.2 \times 10^{4} \mathrm{erg} \mathrm{cm}^{-2} \mathrm{~s}^{-1}$ with wavelengths from 450 to $900 \mathrm{~nm}$. Receptor and horizontal cell responses were identified by the criteria given by Baylor and Fuortes ${ }^{14}$.

The effects of calcium-strontium substitution on the responses to light of a horizontal cell are shown in Fig. 1. Adding $\mathrm{Sr}^{2+}$ to the retinal medium restores the light response of the cell abolished by calcium removal (Fig. 1A). The shape of transients at the light onset and offset, however, differs in the presence of $\mathrm{Sr}^{2+}$ with respect to the control Ringer (Fig. $1 B$ ). Similar changes are observed in the depolarising transient at the light offset, both in strontium and EDTA Ringer, and so they seem to depend on the

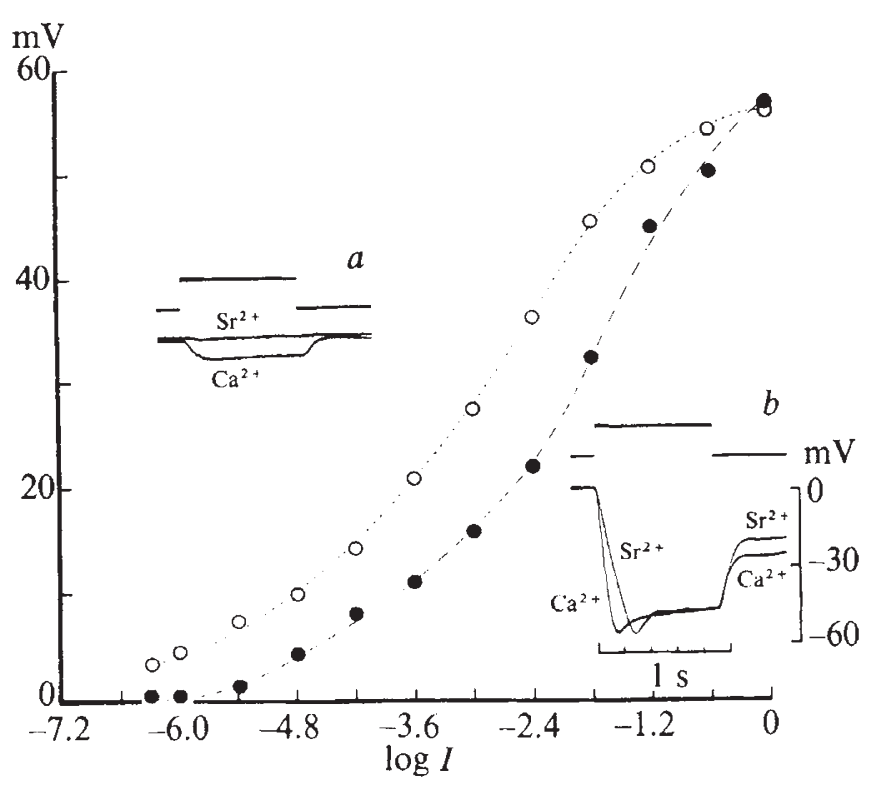

Fig. 2 Peak amplitude of the response of a horizontal cell in control Ringer $(\circlearrowleft)$ and during perfusion with calcium-free strontium solution (O), plotted as a function of light intensity. Records in insets $a$ and $b$ are the responses obtained in the two conditions to light attenuated by $5.4 \mathrm{log}$ units $(a)$ and to the maximum available energy $(b)$. Area illuminated was 1,200 $\mu \mathrm{m}$ in diameter.

absence of calcium ions, while the prolongation of the depolarising phase at the light onset is seen only in the absence of strontium. Further differences between the response to light of horizontal cells in control Ringer and in calcium-free strontium solution, are illustrated in Fig. 2, in which the stimulus response functions $(V / \log I)$ are plotted for the same horizontal cell in the two conditions. The amplitude of the response to light in the presence of $\mathrm{Sr}^{2+}$ is lower than that in control Ringer over most of the intensity range (sec in particular inset $a$ ) but approaches the control amplitude at the highest intensity (see inset $b$ ).

These differences in the response of the horizontal cell can be interpreted as being due, at least in part, to the action of the ionic changes on the responses of presynaptic neurones-that is, cones. Indeed, control experiments have shown that during the perfusion with low calcium-strontium solution the $V / \log I$ curves of the cones and their depolarising transients at the end of the stimulus are modified in a way comparable to that observed in the horizontal cells. Similar modifications are also observed during perfusion with low calcium-EDTA Ringer, again suggesting that they

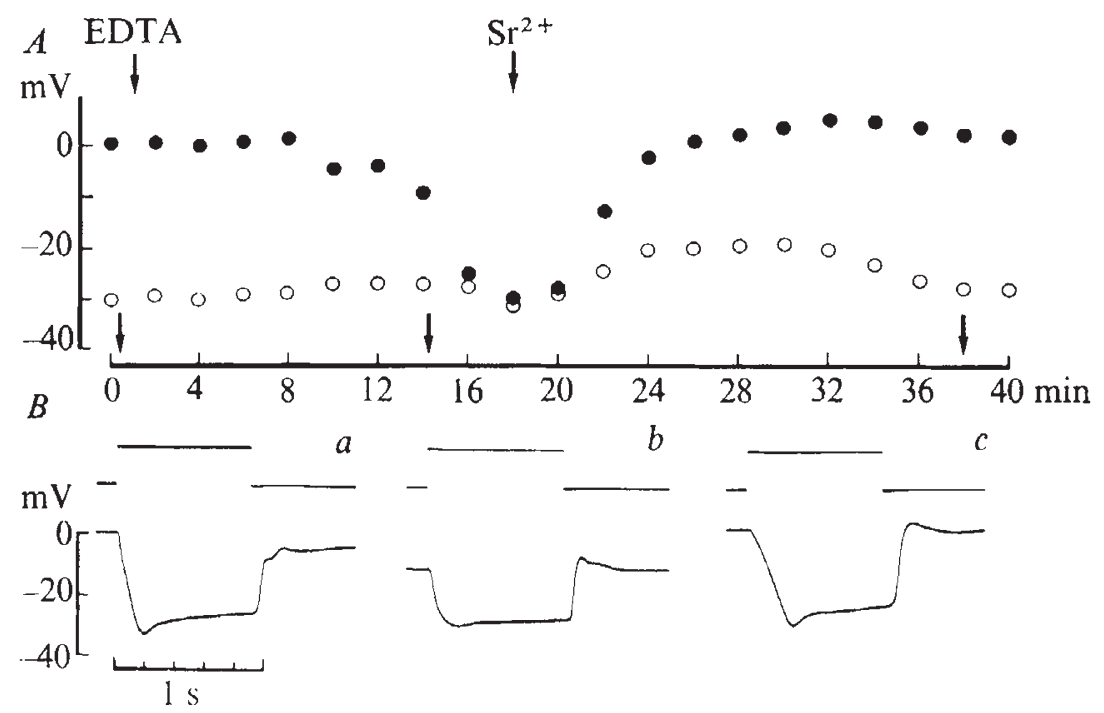

Fig. $1 A$ and $B$, Effects of calcium-strontium substitution on response to light of a horizontal cell. $A,-$, Level of membrane potential in darkness; $O$, peaks of responses to light. $B$, Zero level of membrane potential is arbitrary and indicates the level of membrane potential in darkness during the perfusion with control Ringer. During the experiment the retina was stimulated with long pulses of light (see the trace above each record in $B)$ at a constant rate $\left(0.1 \mathrm{~s}^{-1}\right)$. Light intensity was attenuated by $2.4 \mathrm{log}$ units with respect to the maximum available energy; the area illuminated was 1,200 $\mu \mathrm{m}$ in diameter. Arrows on abscissa in $A$ indicate the time at which the records in $B$ were taken. EDTA indicates the application of a Ringer solution without calcium containing $2 \mathrm{mM}^{-1}$ of EDTA, and $\mathrm{Sr}^{2+}$ the application of a Ringer solution without calcium, containing $2 \mathrm{mM}^{-1}$ of strontium ions. 
depend on the absence of calcium ions from the medium rather than on the presence of strontium (see also ref. 8 ). On the contrary, the time course of the hyperpolarising transient of the cones at the onset of the stimulus is not significantly different in the three conditions.

Previous work has shown that the transmission of electrical signals from photoreceptors to horizontal cells depends critically on the presence of calcium in the extracellular fluid ${ }^{7}$ and is blocked either by excess magnesium ${ }^{6-8}$ or by the presence of cobalt ions ${ }^{7}$. In this paper I have demonstrated that the addition of strontium ions to the retinal medium restores the response of horizontal cells to light abolished by calcium removal. All these properties are characteristic of the chemical transmission in conventional synapses and therefore these results strongly support the chemical nature of the signal transmission from photoreceptors to horizontal cells.

As to the modifications in the time course of the depolarising transient and in the $V / \log I$ curves observed in the response of the horizontal cells during the perfusion with calcium-free strontium Ringer, these seem to depend on similar changes in the behaviour of the cones in the same conditions. On the contrary, the prolongation of the time course of the hyperpolarising onset in the horizontal cells responses has no counterpart in cone responses. At the neuromuscular junction $\mathrm{Sr}^{2+}$ ions are known to increase, by way of a postsynaptic effect, the duration of transmitter action. If one assumes that the hyperpolarising responses to light of horizontal cells result from a reduction in the release of a depolarising transmitter ${ }^{15}$ continuously flowing in darkness from the receptor pedicle, increasing the duration of transmitter action would result in a slower time course of the hyperpolarising phase at the light onset.

I thank Dr L. Cervetto for suggestions and Drs L. Maffei, A. Fiorentini and A. Cangiano for reading the manuscript.

Laboratorio di Neurofisiologia del CNR,

\section{Piccolino}

Pisa, Italy

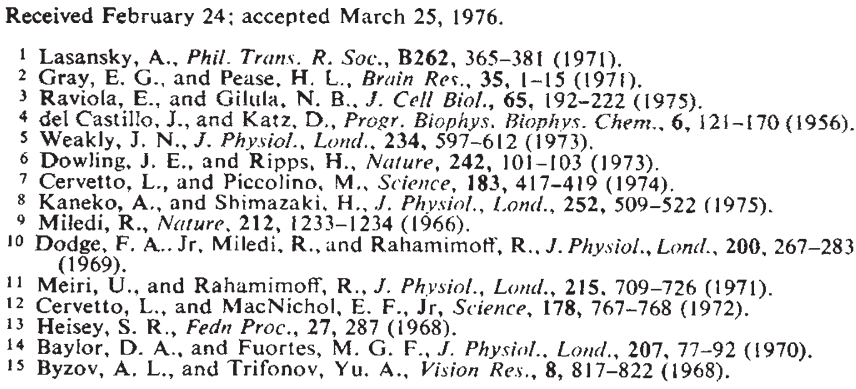

\section{Postexposure serum prophylaxis of neonatal herpes simplex virus infection of mice}

Perinatal infections with herpes simplex virus (HSV) cause death or permanent disability in most reported cases ${ }^{1-5}$. In theory, control of this disease by immunotherapy might be feasible, much like postexposure serum prophylaxis of rabies, since the time of HSV infection in the birth canal is usually known and antibody can be administered soon thereafter. Treatment with antibody has not been encouraged, however, because (1) many investigators consider this immunity cellmediated rather than humoral ${ }^{6-11} ;(2)$ effective administration of human immune globulin in neonatal herpes of man has not been demonstrated ${ }^{1.3,12}$, and (3) transplacental antibody conferred only partial protection in human neonatal infections ${ }^{1}$. There is further discouragement in animal studies which failed to show protection by antibody ${ }^{9-11}$, although antibody was protective in some animal studies ${ }^{13-20}$. The findings reported here raise again the possibility that antibody treatment might be effective in specific conditions.

The concept that cell-mediated immunity, rather than humoral immunity, is the major host defence has been based in part on increased severity of HSV infection after impairment of T-lymphocyte function ${ }^{6-11}$. But, findings that HSV antibody production is $T$-cell dependent ${ }^{9.20 .21}$ suggest that exacerbation of some of these infections by impaired T-lymphocyte function could have been due to decreased antibody formation ${ }^{20}$. Furthermore, protection of mice that received syngeneic, immune spleen cells and were infected with HSV might, in some cases, be due to the transfer of antibody-producing or helper cells, since high levels of antibody were produced more rapidly in these mice than in control mice (unpublished observations). Consistent with this view is the protection of nude ${ }^{20}$, immunosuppressed, and normal mice (unpublished observations) against HSV infection by sufficiently high levels of passively administered antibody and apparent protection against HSV by maternal antibody of infants (excluding newborns) under 11 months of age ${ }^{22}$.

Re-examination of antibody potential in a model of neonatal HSV infection is indicated by these findings, even though they do not establish the importance of antibody in all conditions. For example, strains of HSV that spread principally through the blood stream might be affected by antibody, but not strains of HSV that are transmitted through nerve pathways which exclude antibody.

Our study was designed to determine whether administration of various units of neutralising antibody could achieve postexposure prophylaxis of experimental neonatal HSV infection ${ }^{20}$. Mice (Swiss, CDF-1 or BALB/c), age 1-4 d, were infected subcutaneously with from 1 to 300 newborn mouse LD $_{50}$ of HSV, either type 1 (VR3 strain) or type 2 (MS strain). One hour after infection, the experimental mice were injected intraperitoneally with 0.1 or $0.2 \mathrm{ml}$ of type-specific hyperimmune rabbit antiserum. Controls were injected with normal rabbit or foetal bovine serum, or Eagle's tissue culture medium containing $2 \%$ foetal bovine serum. Mice were observed for at least $12 \mathrm{~d}$. Percentage protection was calculated as follows:

$\frac{\% \text { mortality of controls }-\% \text { mortality of treated }}{\% \text { mortality of controls }}$

Rabbit antisera were prepared by initial injections of HSV virus either type 1 or type 2 (grown in rabbit kidney cultures and emulsified with Freund's incomplete adjuvant) into the footpads and back $\left(0.25 \mathrm{ml}\right.$ containing $10^{5.4}$ PFUs of HSV), followed by four to eight weekly subcutaneous and intravenous injections of virus without adjuvant.

Protection consistently occurred in newborn mice infected with 1-20 LD 50 of either type of HSV and treated with a total of $12,000 \mathrm{U}$ of antibody (Table 1). No protection occurred when the large dose of antibody was administered to mice infected with a high challenge dose $\left(100-300 \mathrm{LD}_{50}\right)$ of HSV. Little or no protection followed treatment with $600 \mathrm{U}$ of antibody. Better results attained against HSV type 1 warrant further study.

Our findings demonstrate good protection against experimental neonatal HSV infection by large doses of antibody in mice infected with low challenge doses of virus. Failure of antibody in some animal studies ${ }^{9-11}$, but not in others ${ }^{13-20}$, could conceivably be due to such variables as antibody dose ${ }^{17}$, virus challenge dose ${ }^{14}$, portal of entry HSV, and difference in pathogenesis, depending on the virus strain used. These same factors might account for the reported failures of human immune globulin to protect newborn infants against HSV nfection ${ }^{1,3,12}$. Additional study of these and other variables may further determine the optimum conditions for antibody protection of newborns.

Although our results were obtained using mice infected subcutaneously, other studies have shown antibody protection 